\title{
A!
}

This is an electronic reprint of the original article.

This reprint may differ from the original in pagination and typographic detail.

Martikainen, J.-P.; Heikkinen, M.O.J.; Törmä, P.

\section{Condensation phenomena in plasmonics}

Published in:

Physical Review A

DOI:

10.1103/PhysRevA.90.053604

Published: 01/11/2014

Document Version

Publisher's PDF, also known as Version of record

Published under the following license:

CC BY-NC

Please cite the original version:

Martikainen, J-P., Heikkinen, M. O. J., \& Törmä, P. (2014). Condensation phenomena in plasmonics. Physical Review A, 90(5), 1-8. [053604]. https://doi.org/10.1103/PhysRevA.90.053604

This material is protected by copyright and other intellectual property rights, and duplication or sale of all or part of any of the repository collections is not permitted, except that material may be duplicated by you for your research use or educational purposes in electronic or print form. You must obtain permission for any other use. Electronic or print copies may not be offered, whether for sale or otherwise to anyone who is not an authorised user. 


\title{
Condensation phenomena in plasmonics
}

\author{
J.-P. Martikainen, ${ }^{*}$ M. O. J. Heikkinen, and P. Törmä ${ }^{\dagger}$ \\ COMP Centre of Excellence, Department of Applied Physics, Aalto University, P.O. Box 15100, Fi-00076 Aalto, Finland
}

(Received 27 June 2014; published 5 November 2014)

\begin{abstract}
We study arrays of plasmonic nanoparticles combined with quantum emitters, quantum plasmonic lattices, as a platform for room-temperature studies of quantum many-body physics. We outline a theory to describe surface plasmon-polariton distributions when they are coupled to externally pumped molecules. The possibility of tailoring the dispersion in plasmonic lattices allows realization of a variety of distributions, including the Bose-Einstein distribution as in photon condensation [Klaers et al., Nature (London) 468, 545 (2010)]. We show that the presence of losses can relax some of the standard dimensionality restrictions for condensation.
\end{abstract}

DOI: 10.1103/PhysRevA.90.053604

\section{INTRODUCTION}

Metallic nanoparticles arranged in periodic arrays display so-called surface lattice resonances (SLRs) with relatively narrow linewidths [1-3]. SLRs offer complete designability of dispersion using nanofabrication techniques, which rivals the freedom of tailoring dispersions in optical lattices [4,5], but now on the nanometer scale. The SLR modes are based on localized surface plasmon resonances (LSPRs) of the nanoparticles and diffractive orders of the lattice. The LSPRs are confined to $10-100 \mathrm{~nm}$ dimensions, providing small mode volumes. Indeed, many-emitter strong coupling has been observed in these and other plasmonics systems [6]: the normal mode splittings (Rabi splittings) are of the order 100-1000 meV, which means that the light-matter hybrids formed by strong coupling exist at room temperature. It has been predicted that strong coupling at the single-emitter level is feasible at room temperature and without external cavities [6]. The effective masses of the hybrids in plasmonic systems range typically from $10^{-8}$ to $10^{-5}$ electron masses, suggesting the possibility of Bose-Einstein condensation at room temperature, for instance (see Appendix A). Thus, we envision that plasmonic lattices combined with emitters, which we call quantum plasmonic lattices, can become a platform for quantum many-body physics at room temperature and on chip. Such similar concepts as the superfluid-Mott-insulator transition in optical lattices [7,8], exciton-polariton condensates [9-11], light condensation [12], thresholdless lasing [13,14], and quantum fluids of light in general [15] (among others) can be explored in quantum plasmonic lattices. Initial results on lasing in similar systems have already been reported [16-18].

The role and design of interactions is a challenge and an opportunity in quantum plasmonic lattices. Photon-photon interactions, mediated via either strong-coupling hybridization or weak-coupling interaction with the quantum emitters, are of a different nature than in the ultracold gas, semiconductor, and microcavity systems mentioned above. Another difference is the highly lossy character of SLR modes (10-100 fs lifetimes generally), although with SLRs the lifetimes can be potentially increased to 100-1000 fs. A short lifetime is connected to the desirable feature of ultrafast operation speed, but it may hinder interesting quantum and coherence phenomena. In this article,

\footnotetext{
*jani-petri.martikainen@aalto.fi

†paivi.torma@aalto.fi
}

PACS number(s): 67.85.Hj, 33.80.-b, 05.30.-d, 73.20.Mf

as a primary example of the physics in quantum plasmonic lattices, we investigate the possibility of condensation of surface plasmon polaritons (SPPs) in the SLR modes. We show that by tailoring the SPP losses and dispersions different SPP distributions, including one resembling the Bose-Einstein distribution, can be realized.

\section{PHYSICAL MODEL}

We ask whether SPPs supported by the SLR modes, interacting with quantum emitters in the weak-coupling regime, can display well-defined statistical distributions including cases that show condensation-even in the presence of high losses. We consider a system of metallic nanoparticle arrays supporting SLRs; see Fig. 1. The nanoparticles are anisotropic so that the SLR dispersions are effectively one dimensional. On top of the metallic array structure, we assume a number of quantum emitters that have a two-level structure where the levels are split into multiple sublevels; in practice the emitters can be dye molecules embedded in a polymer matrix, for example, with the electronic levels split into a rovibrationallevel substructure. Under appropriate conditions, plasmonic modes can strongly couple to molecules [19-21]; here, we consider only the weak-coupling regime. Therefore, we do not consider the possibility of condensation of light-matter hybrids in analogy to semiconductor exciton-polariton condensates $[9,10]$ but phenomena more similar to the concept of photon condensation [12,22,23].

We consider molecules pumped so that a certain fraction of them are on the excited-state manifold. This implies that a loss-compensating pump is implicit in our model. Molecular manifolds thermalize at picosecond time scales due to their coupling to the phonon bath of the polymer so that both excited- and ground-state rovibrational manifolds are separately assumed to be in equilibrium Maxwell-Boltzmann distributions corresponding to the sample temperature. Relaxation from the excited to the ground-state is assumed to occur on the time scale of nanoseconds. We assume the molecular decay is dominated by the coupling to the plasmonic modes, that is, a large branching ratio $\beta_{b}$ between spontaneous emission to the modes of interest versus other modes [14,24]. This is justified in plasmonic systems where both enhanced spontaneous emission, that is, the Purcell effect [25], and strong coupling can be easily achieved $[6,17]$. Although $\beta_{b}$ is large, we are not working in the limit of thresholdless lasing [14] (see Appendix B). 

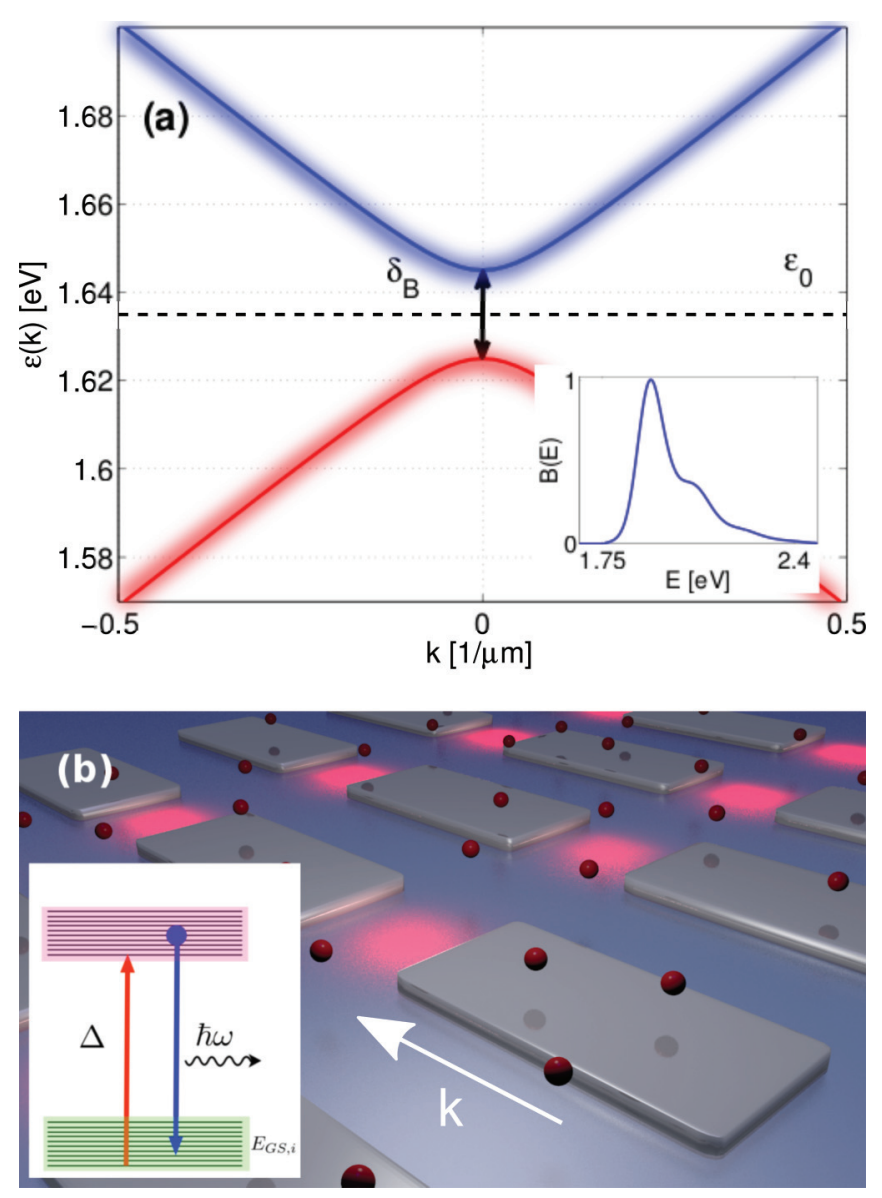

FIG. 1. (Color online) SLR dispersions as a function of wave number $k$ in the plane of the nanoparticle array. Here we choose $\epsilon(k)=\hbar \omega(k)= \pm \sqrt{(\hbar c k / n)^{2}+\left(\delta_{B} / 2\right)^{2}}+\epsilon_{0}$, where $n=1.51$ is the index of refraction, $\delta_{B}=10 \mathrm{meV}$ is a gap between branches, and $\epsilon_{0}$ is the energy offset. (a) shows the two SLR branches so that the blurred line on the background indicates finite width of the SLR. In the inset we show the absorption profile $B(\omega)$ for the DiD molecule at $E=\hbar \omega$ based on the manufacturer's data together with a linear extrapolation close to the absorption edge. (b) shows a schematic description of the system with rectangular nanoparticles, emitters (red balls), spatially varying electromagnetic field strength, and a simplified model of the molecule energy-level structure in the inset, with ground-state levels $E_{\mathrm{GS}, i}\left(E_{\mathrm{GS}, 1}, E_{\mathrm{GS}, 2}, \ldots\right)$. The white arrow indicates the direction of the wave vector where dispersion varies.

In our computations, we consider dye molecules, although any emitters with a similar level structure would do. We take the absorption properties of the dye molecules to be those of DiD molecules (as used in our experiments in [21]). Importantly (based on the manufacturer's data) the emission profile is well predicted by the Kennard-Stepanov relation, proportional to $B(\omega) e^{-\beta(\hbar \omega-\Delta)}$ at room temperature $\left(\beta=1 / k_{B} T\right)$. Here $\Delta$ is the difference between the lowest excited-manifold energy and the ground-state energy, $\hbar \omega$ is the SPP energy, and $B(\omega)$ is the absorption profile (see Fig. 1). In the limit where the ground-state manifold is narrow, $\Delta$ approaches the absorption edge of the molecules. We model the molecules as systems with ground- and excited-state manifolds that have a rovibrational level structure; see Fig. 1. We take this level structure to be ten equidistant energy levels so that the minimum separation of the manifolds corresponds to the absorption edge. While realistic molecules are more complicated, these assumptions do not change the expected qualitative behavior strongly. The molecules are assumed to be embedded in a medium, for example, a polymer matrix.

\section{RATE-EQUATION MODEL}

Generally the time evolution of a quantum system can be written in terms of a master equation for the density matrix. A simplified description can be obtained by neglecting off-diagonal coherence terms and focusing on populations appearing on the diagonal. These follow rate equations based on transition rates between states [26,27].

Let us explore the evolution of one $k$ mode [corresponding to a SPP energy of $\hbar \omega(k)]$ with occupation $n$. This mode can decay at a rate $\Gamma$, and this gives rise to a loss term $\mathcal{L}$ in the rate equation,

$$
\mathcal{L}=-\Gamma n .
$$

A SPP may also be absorbed by the dye at a rate $R_{\mathrm{abs}}$, and since there are many transitions and the absorption rate depends on the absorption profile $B$ of the dye, we get an absorption term

$$
\mathcal{A}=-B M R_{\mathrm{abs}} n,
$$

where $M=\sum_{i} \exp \left(-\beta E_{\mathrm{GS}, i}\right)$ is a factor that accounts for the molecular levels in thermal equilibrium. Here we assumed equidistant (splitting of $\sim 10 \mathrm{meV}$ ) molecular rovibrational levels over a range of $\sim 100 \mathrm{meV}$, but in the end this factor depends on the details of the molecular-level structure. Note that in Ref. [28], many molecular transitions can contribute to the same photon energy. This was accounted for by an integration over ground-state energies with some rovibrational densities of state. In that case, the steady state could be solved by demanding a vanishing integrand without specifying the densities of states. To find a steady-state solution in our case, the molecules must be specified in greater detail, since the absorption and emission terms must be balanced with the plasmon loss term outside the summation that was absent in Ref. [28]. Furthermore, we assume that the molecular absorption profile depends on the ground-state manifold energy $E_{\mathrm{GS}, i}$ only weakly so that $B\left(\omega, E_{\mathrm{GS}, i}\right) \simeq B(\omega)$.

Finally, molecules can decay into SPPs and this process acts as a source of SPPs. The rate coefficients for spontaneous and stimulated emissions are the same. This similarity together, with the assumption of the Kennard-Stepanov law relating emission and absorption rates and the $M$ factor mentioned earlier, gives a term

$$
\mathcal{E}=M B R_{\text {spon }}(1+n) e^{-\beta[\hbar \omega(k)-\Delta]} .
$$

The ratio of $R_{\text {spon }}$ and $R_{\text {abs }}$ depends on the number of excitedand ground-state molecules, as will be discussed below.

As a result of these considerations SPP occupations $n(k)$ are described by a rate equation

$$
\begin{aligned}
\dot{n}(k)= & -\Gamma(\omega(k)) n(k)+\sum_{i} B(\omega(k)) e^{-\beta E_{G S, i}} \\
& \times\left[R_{\mathrm{spon}}(\omega(k))[1+n(k)] e^{-\beta[\hbar \omega(k)-\Delta]}\right. \\
& \left.-R_{\mathrm{abs}}(\omega(k)) n(k)\right] .
\end{aligned}
$$


Here, $\omega(k)$ is the SLR dispersion. The summation is over ground-state molecular manifold energy levels $E_{\mathrm{GS}, i}$. We estimate the SPP decay rate $\Gamma(\omega)$ from our experiments [21]. With reasonable accuracy it can be taken as a constant of magnitude $\Gamma(\omega)=10 \mathrm{meV}$. This term, addressing the short lifetimes of SPPs, is one of the essential differences when compared to previous studies of photon condensation in microcavity systems [28] where such a term was ignored due to longer lifetimes. The remaining terms describe spontaneous and stimulated emissions at rate $R_{\text {spon }}(\omega)$ from molecules to SPPs as well as absorption at the rate $R_{\mathrm{abs}}(\omega)$. Note that chemical potential does not appear in the rate equation since the SPP number is not conserved.

The number of molecules $N_{M}$ coupled to the SPPs should not be obtained from the total number of molecules in the sample, but instead as the number of molecules within an effective mode volume; this is estimated (see Appendix C) to be around $N_{M}=24 \times 10^{6}$ dye molecules in our system. We define $N_{M}=N_{e}+N_{g}$, where $N_{e}$ is the number of molecules in the excited manifold and $N_{g}$ corresponds to the molecules in the ground state.

The rates are affected by the number of excited-state molecules $N_{e}$ relative to ground-state molecules $N_{g}$, and the quantum yield $\Phi$ of the dye molecule. We take the rate coefficient for the spontaneous (and stimulated) emissions to be

$$
R_{\text {spon }}(\omega)=\frac{F}{\tau} N_{e},
$$

where $\tau$ is the characteristic lifetime of the excited-state molecule in vacuum, chosen as $\sim 5 \mathrm{~ns}$. In principle, the spontaneous emission rate in free space depends on energy as proportional to $\omega^{d}$ ( $d$ is the dimensionality), but we ignore the energy dependence here since the range of energies where distributions vary is small compared to absolute photon energies and the presence of metal will change such dependencies. Furthermore, $F$ is the Purcell factor and describes the enhanced decay rate of the molecules close to plasmonic structures. Interestingly, large Purcell enhancement will allow smaller population inversions, that is, smaller $N_{e}$. Since $F$ is unknown and depends on experimental details, we choose $F=20$ in our computations so that it is likely of correct order of magnitude with respect to the range of reported values from $F=6$ to $F=200[16,17]$. The absorption rate coefficient is taken as

$$
R_{\text {abs }}(\omega)=N_{g} R_{\text {spon }}(\omega) /\left(N_{e} \Phi\right) .
$$

Note that since the total absorption rate scales with the number of molecules, the rate is, in our case, typically a few orders of magnitude larger than the SPP decay rate.

The SPPs with energies in the $2 \mathrm{eV}$ range are well separated from thermal excitations at $k_{B} T=26 \mathrm{meV}$, so the only way to excite them is via decay from molecules. The SPPs can have fairly complicated dispersions, and their widths can vary, for example, as a function of the photon momenta in the plane of the nanoparticles. The dispersions are typically nearly linear; however, a band gap can be designed by breaking the symmetry of the array; see Fig. 1. It should be emphasized that SLR dispersions can be tailored at will with different structures, and our choices are therefore only indicative of the physically possible dispersions. The anisotropy of the array implies that SPPs of only one polarization state play a role here, and the dispersion is essentially one dimensional [29]. The SLR dispersion used here is, as given in the caption of Fig. 1,

$$
\epsilon(k)= \pm \sqrt{(\hbar c k / n)^{2}+\left(\delta_{B} / 2\right)^{2}}+\epsilon_{0} .
$$

Here $k$ is the wave number in the plane of the nanoparticle array, $n$ is the index of refraction, $\delta_{B}$ is the gap between the upper and lower SLR branches, and $\epsilon_{0}$ is located in the middle of the dispersions. We use the typical values $n=1.51$ and $\delta_{B}=10 \mathrm{meV}$, and $\epsilon_{0}$ is varied.

\section{STEADY-STATE SOLUTION}

The rate equations have a steady-state solution

$$
n(k)=\frac{1}{g(\omega) e^{\beta(\hbar \omega-\Delta)}-1},
$$

where we defined

$$
g(\omega(k))=\left[M B(\omega) R_{\mathrm{abs}}+\Gamma(\omega)\right] /\left[M R_{\mathrm{spon}} B(\omega)\right]
$$

and $M=\sum_{i} \exp \left(-\beta E_{\mathrm{GS}, i}\right)$. Now $k$ states for which the denominator (nearly) vanishes may have, for certain parameters, diverging populations compared to the other $k$ states, i.e., macroscopic occupation. If the term $g(\omega) e^{\beta(\hbar \omega-\Delta)}$ is much larger than unity, then a distribution similar to the MaxwellBoltzmann one is obtained. To have diverging populations of the lowest states, one should have $g(\omega) e^{\beta(\hbar \omega-\Delta)}$ around 1 for small energies. This implies $R_{\text {abs }} / R_{\text {spon }}=N_{g} /\left(N_{e} \Phi\right) \sim 1$. Note that if molecules experience losses to other modes than SLRs, these could be taken into account by reducing the quantum yield $\Phi$.

To have the term -1 in the denominator of Eq. (8), the term $n(k)$ in $[1+n(k)]$ in the rate equation (4) is needed: this means having stimulated emission into the mode of interest. Bosonic enhancement is thus behind any SPP condensation with statistics deviating from the Maxwell-Boltzmann distribution. The spontaneous emission term, the unity in $[1+n(k)]$, is needed to have a nonzero SPP number, since there is no direct pumping into the mode (see Appendix B). Losses play a role somewhat similar to absorption. In the limit of small losses $(\Gamma)$ and absorption ( $\left.R_{\mathrm{abs}}\right)$, the distribution (5) becomes negative, meaning there is no steady state and only exponentially growing solutions; we differentiate condensation from lasing by exponentially growing $n$, that is, a positive gain coefficient for the latter. In Fig. 2, we demonstrate the distributions for realistic experimental parameters.

How close to a Bose-Einstein distribution can one make the distribution? By moving the function $g(\omega)$ in Eq. (5) into the exponent we find a distribution with the "chemical potential" $\mu(\omega)=\Delta-\frac{1}{\beta} \ln g(\omega)$ that generally depends on energy. In the special case where losses disappear, $\Gamma(\omega)=0$, the result becomes similar to that for the chemical potential of photons in a photon BEC [28] where the photon fugacity was essentially fixed by the excitation level of the system. More generally it should be noted that when $\Gamma(\omega) / B(\omega)$ is constant [the other quantities in Eq. (3) are assumed constant] $\mu(\omega)$ is also a constant and the distribution still looks like a Bose-Einstein distribution even in a lossy environment. This is interesting considering the freedom of tailoring the dispersion 

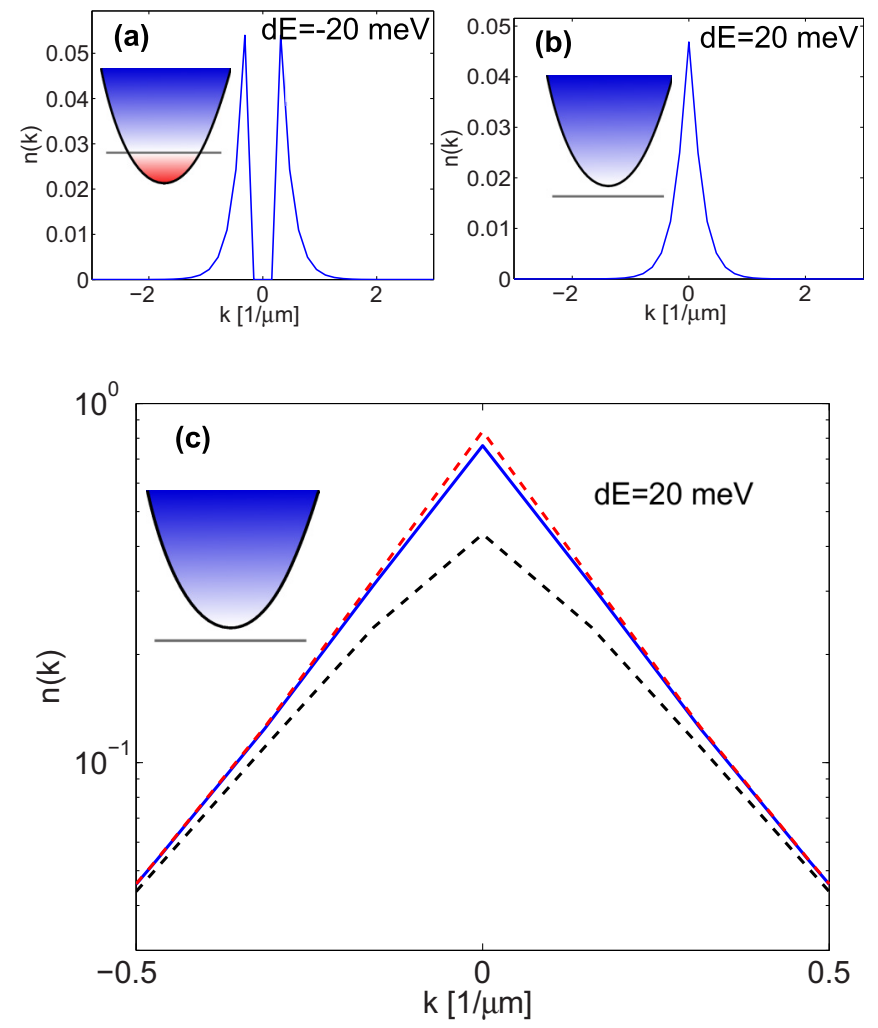

FIG. 2. (Color online) SPP occupation numbers in the upper branch at room temperature as a function of in-plane momentum when losses were $\Gamma(\omega)=10 \mathrm{meV}$. Depending on the relative positions of the molecule absorption edge and the dispersion band edge, one observes accumulation of population to different $k$ states. In (a) and (b) we choose $N_{e} / N_{g}=0.1$ and different figures correspond to different separations of the absorption edge of the molecules from the minimum of the SLR dispersion, $d E=\min [\hbar \omega(k)]-\Delta$. The hole in the first distribution is caused by the absorption edge of the molecules being at higher energy than the minimum of the SLR dispersion. The position of the absorption edge is indicated schematically in the insets by the gray line. Only modes in the blue region are appreciably pumped by molecules. As the temperature increases this feature becomes less sharp. In (c) we approached inversion so that $N_{e} / N_{g}=0.99$. The solid line is from our theory, the dashed black line shows the Maxwell-Boltzmann result without " -1 " in the denominator of the distribution, and the dashed red line is with a Bose-Einstein distribution where $g(\omega)$ was evaluated close to the absorption peak at $1.92 \mathrm{eV}$.

in plasmonic lattices. Like dispersions, loss profiles can also be designed by the lattice geometry. Therefore, it is possible to engineer loss profiles to match the absorption profile of the emitters, and thus perhaps guarantee a constant $\Gamma(\omega) / B(\omega)$. One possibility for doing this is to couple the SLR of interest to another resonance (e.g., caused by another diffractive order in the lattice) so weakly that no splitting but merely a broadening of the dispersion is caused near the desired location in energy.

Furthermore, a Bose-Einstein-like distribution might appear over some energy intervals but not in others. In Fig. 3 we show an example of how the function $g(\omega)$ behaves when the loss coefficient is constant $[\Gamma(\omega)=10 \mathrm{meV}]$ and the absorption profile corresponds to that of the DiD molecule.

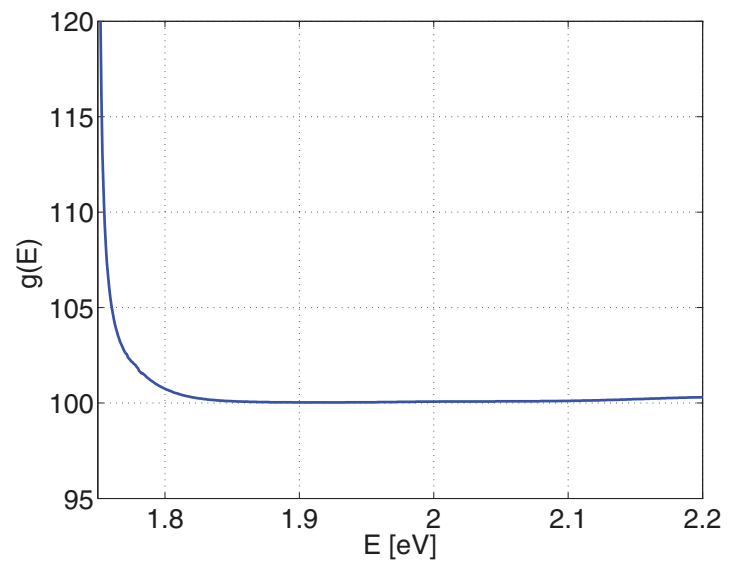

FIG. 3. (Color online) The function $g(\omega)$ in our example system with $N_{e} / N_{g}=0.01(E=\hbar \omega)$. For the $\mathrm{DiD}$ dye molecules the absorption edge is at $\Delta=1.75 \mathrm{eV}$ and we choose $\Gamma(\omega)=10 \mathrm{meV}$, which is a reasonable estimate based on experiments [21]. For a Bose-Einstein distribution this function should be constant.

As can be seen, this function is almost constant over a broad range and has strong energy dependence only close to the absorption edge where the denominator of $g(\omega)$ approaches zero. Beyond this regime, around the absorption peak, $g(\omega)$ is almost constant for an energy range much broader than room temperature; it is in this range where the Bose-Einstein (BE) distribution may be expected even without special tailoring of the loss profile, as shown in Fig. 2. The condition for diverging populations is

$$
N_{e} \Phi / N_{g} \geqslant\left\{1-\Gamma(\omega) \tau /\left[M F N_{e} B(\omega)\right]\right\}^{-1} .
$$

As mentioned earlier, in the case of a constant $\Gamma(\omega) / B(\omega)$ the resulting distribution is the $\mathrm{BE}$ one; otherwise it deviates from the BE distribution, but there is nevertheless divergence of population. We demonstrate the way the excitation fraction required for diverging occupation behaves as a function of quantum yield and loss rate in Fig. 4. With our parameters this quantity depends only relatively weakly on the SPP loss coefficient while the dependence on quantum yield is stronger.

The theory of ideal Bose-Einstein condensates (BECs) with dispersions linear in $k$ shows that a BEC cannot occur in a one-dimensional system. Likewise it is not possible with freeparticle-like $k^{2}$ dispersions in dimensions less than or equal to 2 [30]. In our context, such limitations can be relaxed since the loss profile and the absorption profile both appear in the steadystate solution. To illustrate this, assume $\epsilon=\hbar \omega=\hbar c k / n$. We can then write the total steady-state SPP number ( $L$ is the length of the system)

$$
N_{\mathrm{SPP}}=\sum_{k} n(k)=\frac{L}{2 \pi} \int d k n(k)=\frac{n L}{h c} \int \frac{d \epsilon}{g(\epsilon) e^{\beta(\epsilon-\Delta)}-1}
$$

also in terms of the effective density of states as

$$
N_{\mathrm{SPP}}=\int d \epsilon \frac{\rho(\epsilon)}{e^{\beta(\epsilon-\Delta)}-1},
$$




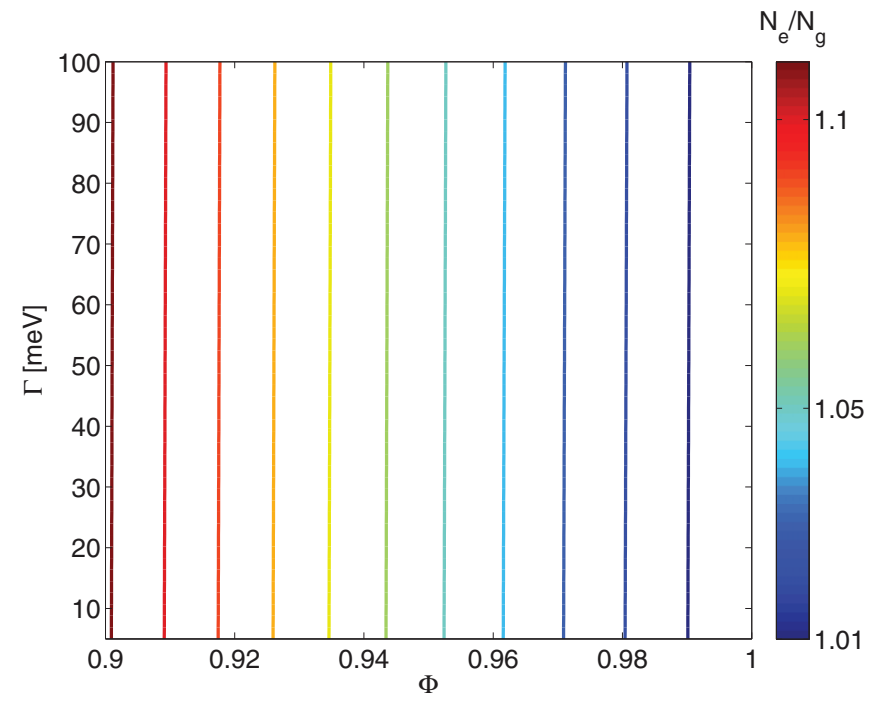

FIG. 4. (Color online) The value of $N_{e} / N_{g}$ required for having diverging SPP occupation number. We kept other parameters the same as elsewhere, but allowed for changes in the quantum yield $\Phi$ of the emitter and the loss rate $\Gamma$. (The energy was fixed at $1.92 \mathrm{eV}$ where the energy dependence of the absorption profile is weak.)

where

$$
\rho(\epsilon)=\frac{n L}{h c} \frac{e^{\beta(\epsilon-\Delta)}-1}{g(\epsilon) e^{\beta(\epsilon-\Delta)}-1} .
$$

This function is not the physical density of states, and consequently BEC-like distributions can appear even in lowerdimensional systems if the loss behavior is appropriate. In this case close to the absorption edge, $\rho(\epsilon)$ vanishes like the density of states for a three-dimensional photon gas or for massive particles in a three-dimensional harmonic trap (see Appendix D). The integral over energy is therefore well behaved in a similar way as in the theory of the ideal BEC.

\section{LASING}

Finally, we connect the results to lasing in these systems. For exponentially growing solutions of Eq. (4) to occur, the gain coefficient

$$
\alpha(\omega)=\frac{M B(\omega)\left[R_{\text {spon }}(\omega) e^{-\beta(\hbar \omega-\Delta)}-R_{\text {abs }}(\omega)\right]}{\Gamma(\omega)}-1
$$

should be positive. This is possible only if

$$
\frac{N_{e} \Phi}{N_{g}}>e^{\beta \hbar(\omega-\Delta)}
$$

which implies population inversion. This is not a sufficient condition for positive gain. While close to the absorption edge, the exponential approaches 1 ; the $B(\omega)$ multiplier in the gain coefficient approaches zero.

In Fig. 5, we demonstrate the behavior of the maximum of the gain coefficient. The parameters of the experiment [17] that reported an observation of lasing in plasmonic arrays correspond roughly to the left corner of Fig. 5. In our example, the maximum gain there is indeed positive $(\alpha \approx 2.4)$, which means that our model is consistent with existing experiments.

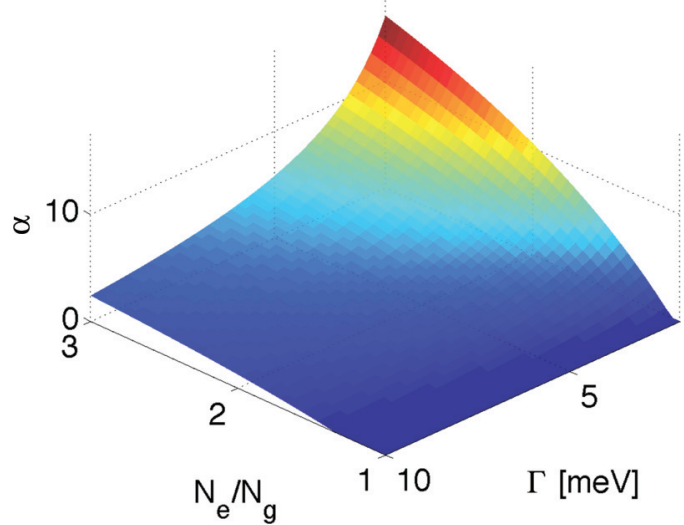

FIG. 5. (Color online) The maximum of the gain coefficient $\alpha(\omega)$ as a function of inversion $N_{e} / N_{g}$ and the loss rate. We choose $\Delta=$ $1.75 \mathrm{eV}$, temperature $T=300 \mathrm{~K}$, and a Purcell factor of $F=20$.

\section{CONCLUSIONS}

We have shown that condensation phenomena are possible even in high-loss-rate systems, such as plasmonic lattices combined with emitters. Population inversions of $N_{g} /\left(N_{e} \Phi\right) \sim 1$ are needed to achieve the regime where the distribution deviates from the Maxwell-Boltzmann one and may show diverging occupation numbers for certain states. By tailoring the dispersions and loss profiles, different types of distribution can be produced, including the Bose-Einstein distribution. Coherence and quantum statistical properties of these systems should be characterized in future work, including higherorder correlations beyond the rate-equation approach [31,32]. Condensation of SPPs may be the way to produce highly coherent light in nanoscale with low powers and modest population inversions.

\section{ACKNOWLEDGMENTS}

We thank Jildou Baarsma, Tommi Hakala, Robert Moerland, Heikki Rekola, and Lei Shi for useful discussions. This work was supported by the Academy of Finland through its Centres of Excellence Program (Projects No. 251748, No. 263347, and No. 13272490) and by the European Research Council (Grant No. ERC-2013-AdG-340748-CODE)

\section{APPENDIX A: ESTIMATES FOR THE EFFECTIVE MASS}

The interplay between diffraction orders, localized surface plasmon resonances, and emitters may introduce splittings of the surface lattice resonances. In the absence of coupling to emitters, surface lattice resonances can have (for example) a dispersion of type

$$
\epsilon(k)=\epsilon_{0} \pm \sqrt{(\hbar c k / n)^{2}+\left(\delta_{B} / 2\right)^{2}},
$$

where $n$ is the index of refraction, $\delta_{B}$ is a gap between the branches, and $\epsilon_{0}$ is the energy offset. For small wave vectors $k$ in the plane of the nanoparticles, we can use a Taylor expansion of the square root and find a particlelike dispersion that is described by an effective mass

$$
m_{\mathrm{eff}}=\frac{n^{2} \delta_{B}}{2 c^{2}} .
$$


When the splitting is in the range from 10 to $1000 \mathrm{meV}$, the effective mass is between $10^{-8} m_{e}$ and $10^{-6} m_{e}$, where $m_{e}$ is the electron mass. Since, for example, the critical temperature for Bose-Einstein condensation is inversely proportional to particle mass $\left(k_{B} T_{c} \approx 3.3 \hbar^{2} n_{B}^{2 / 3} / m_{\text {eff }}\right.$ for a free Bose gas in three dimensions with density $n_{B}$ ), these low values suggest a possibility for much higher temperatures for phase transitions. However, the above approximation is not valid for all wave vectors, and real dispersions should be used in actual calculations. Expansion is appropriate if $k \ll n \delta_{B} /(2 \hbar c)$ where the right-hand side varies $(n=1.5)$ between $0.04 / \mu \mathrm{m}$ and $4 / \mu \mathrm{m}$.

We can also estimate the effective mass where the above dispersions couple strongly with a molecular energy level of the emitter [20,21]. In this case, the eigenmodes follow from the Hamiltonian

$$
H=\left(\begin{array}{cc}
\sqrt{(\hbar c k / n)^{2}+\left(\delta_{B} / 2\right)^{2}} & \Omega \\
\Omega^{*} & E_{m}
\end{array}\right),
$$

where $E_{m}$ corresponds to the molecular transition energy and $\Omega$ is the coupling between molecules and the surface lattice resonance (without a loss of generality, we choose $\epsilon_{0}=0$ in this case). Solving for the eigenmodes and expanding again around $k=0$ gives us the effective mass

$$
m_{\mathrm{eff}}=\frac{n^{2} \delta_{B} \sqrt{\left(2 E_{m}-\delta_{B}\right)^{2}+16|\Omega|^{2}}}{c^{2}\left[2 E_{m}-\delta_{B}+\sqrt{\left(2 E_{m}-\delta_{B}\right)^{2}+16|\Omega|^{2}}\right]} .
$$

This approaches the previous result as coupling disappears, while in the limit of very large coupling, we have $m_{\mathrm{eff}} \approx$ $n^{2} \delta_{B} / c^{2}$. The effective mass of the hybridized mode is nevertheless always larger than that of the surface lattice resonance. If the splittings in SLR range from $\delta_{B}=$ $10 \mathrm{meV}$ to $\delta_{B}=1000 \mathrm{meV}$ and the Rabi splitting is $100 \mathrm{meV}$ [21], the effective mass varies between $m_{\text {eff }} \sim 10^{-8} m_{e}$ and $m_{\mathrm{eff}} \sim 10^{-5} m_{e}$.

\section{APPENDIX B: COMPARISON TO CAVITY-QED LASER RATE EQUATIONS}

It is instructive to compare the rate equation (5) to the description of a cavity-QED laser [14]. The rate equation of the cavity-QED system in the presence of background absorption is

$$
\begin{aligned}
& \gamma^{-1} \dot{n}=-\lambda n+\beta_{b} n\left(N-N_{0}\right)+\beta N, \\
& \gamma^{-1} \dot{N}=-N+P-\beta_{b} n\left(N-N_{0}\right) .
\end{aligned}
$$

Here, $n$ is the photon number in the cavity mode and $N$ is the number of atoms in the upper level of the lasing transition, in short, the number of carriers. The coefficients $\lambda$ and $P$ are the cavity decay rate and the pumping rate, respectively, measured in units of the spontaneous emission rate $\gamma$. The branching ratio $\beta_{b}$ indicates the fraction of spontaneous emission to the laser mode [24]. Finally, $N_{0}$ is the carrier number at transparency and accounts for the absorption of photons to the medium (more precisely, when $N=N_{0}$, the stimulated emission from and absorption to the medium are equal).

The pair of equations above assumes that the system is pumped with a constant rate $P$. This assumption is the important difference in the rate equation (5) where, conversely, it was assumed that the excited molecular states are in thermal equilibrium with the environment, and that the time scale of equilibration is fast in comparison to the time scale of the rate equation. This is equivalent to replacing the time evolution of the carrier density of the cavity-QED system with an externally determined, constant carrier density $N_{c}$. Incorporating this assumption, only the photon-number rate equation is relevant to the problem. We rewrite this equation as

$$
\dot{n}=-\gamma \lambda n+\gamma \beta_{b} N_{c}(1+n)-\gamma \beta_{b} N_{0} n
$$

We may then readily identify a one-to-one correspondence with the SPP rate equation. The coefficients of the two rate equations map onto each other as follows:

$$
\begin{aligned}
& \gamma \lambda \leftrightarrow \Gamma(\omega(k)) \\
& \gamma \beta_{b} N_{c} \leftrightarrow\left(\sum_{i} B(\omega(k)) e^{-\beta E_{\mathrm{GS}, i}}\right) R_{\mathrm{spon}}(\omega(k)) e^{-\beta[\hbar \omega(k)-\Delta]} \\
& \gamma \beta_{b} N_{0} \leftrightarrow\left(\sum_{i} B(\omega(k)) e^{-\beta E_{G S, i}}\right) R_{\mathrm{abs}}(\omega(k))
\end{aligned}
$$

In other words, the cavity decay rate corresponds to the SPP decay rate, the total emission rate to the cavity corresponds to the total emission rate to the SLR mode, and the background absorption rate corresponds to the absorption of SPP by the molecules. The difference is that the rate equation for the SPP system explicitly takes into account that there are many molecular energy levels which take part in the emission and absorption processes, that is, there is a summation over the molecular energy levels $E_{\mathrm{GS}, i}$.

There are a number of important points to note. Several SLR modes are labeled by the momentum $k$, each of which requires its own rate equation. However, these rate equations are mutually independent since the molecular states are maintained at thermal equilibrium with constant occupation number. Therefore, the mapping to the cavity-QED system is possible on the level of a single SLR mode. The high-loss-rate character of our system guarantees a small SPP number compared to the number of excited molecules; therefore, the assumption of a constant occupation number is reasonable. Thus, although the $\beta_{b}$ factor in our case is large, that is, most of the spontaneous emission is guided to the modes of interest, we are not working in the regime of thresholdless lasing. Our system rather corresponds to the bad-cavity limit. Indeed, if the molecular occupation numbers were time dependent and had feedback from the SPP spectrum, all of the SLR modes would be coupled with each other by the time evolution of the molecular states. In summary, the differences of our case in comparison to the cavity-QED system description [14] are (1) a constant excited-state (carrier density) population is assumed instead of excited-state dynamics, which is justifiable in our high-loss-rate system, (2) we consider several modes, and (3) the excited-state populations and absorption and emission coefficients are temperature and energy dependent. Due to these differences, phenomena distinct from (thresholdless) lasing can be predicted. 


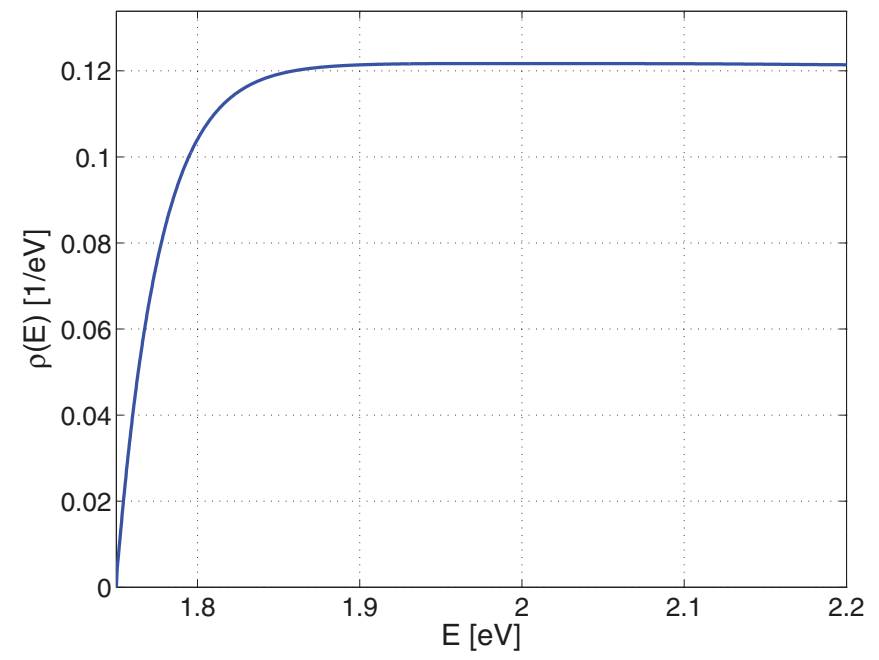

FIG. 6. (Color online) Function $\rho(E)$ with linear SLR dispersions which cross at the absorption edge $\Delta$ of the molecules. We used $\Delta=1.75 \mathrm{eV}, T=300 \mathrm{~K}$, and refractive index $n=1.51$. (For concreteness we choose the length scale in the prefactor in Eq. (D2) to be $L=10 \mu \mathrm{m}$.)

\section{APPENDIX C: ESTIMATE OF THE MOLECULE NUMBER}

The rates in the rate equations are affected-among other things - by the total number of molecules $N_{T}$. The number of molecules $N_{M}$ coupled to the SPPs will, however, be different from the actual number of molecules throughout the sample for several reasons. First, the mode structure of the SLR modes has a strong spatial variation at a length scale of the metallic nanoparticle array. This means that the molecules around "hot spots" dominate the coupling to SPPs. The fraction of such molecules is roughly proportional to the fraction of the surface covered by metal, and in our typical experiments [21] this varies between $0.3 \%$ and $1.3 \%$. Second, the intrinsic losses in the system limit how far the modes can propagate, and coherence lengths related to this are in the range of $\sim 5 \mu \mathrm{m}$ [21], which is much smaller than the dimensions of the nanoparticle array. Together, this suggests that $N_{M}$ should range from $0.1 \%$ to $1 \%$ of the total number of dye molecules. We choose an estimate of $1 \%$.

We consider a molecule concentration of $50 \mathrm{mM}$, and $50 \mathrm{~nm}$ as the thickness of the molecule layer. For a $40 \times$ $40 \mu \mathrm{m}^{2}$ sample, this implies around $N_{M}=24 \times 10^{6}$ dye molecules. This is a reasonable value based on our experiments [21] where the dye concentration ranges from 0 to $800 \mathrm{mM}$, and up to $1 \%$ of the structure was covered by metal.

\section{APPENDIX D: EFFECTIVE DENSITY OF STATES}

As pointed out in the main text, with linear dispersion we can write the plasmon number in terms of the effective density of states as

$$
N_{p l}=\int d \epsilon \frac{\rho(\epsilon)}{e^{\beta(\epsilon-\Delta)}-1},
$$

where

$$
\rho(\epsilon)=\frac{n L}{h c} \frac{e^{\beta(\epsilon-\Delta)}-1}{g(\epsilon) e^{\beta(\epsilon-\Delta)}-1} .
$$

Close to the absorption edge we can take $B(\epsilon) \propto(\epsilon-\Delta)$. Since the loss coefficient $\Gamma(\omega)$ is nonzero and expected to be well behaved at the absorption edge, this implies $g(\epsilon)=$ $a+b /(\epsilon-\Delta)$ (with $a$ and $b$ some positive coefficients) in the vicinity of the absorption edge. Therefore, by expanding $\rho(\epsilon)$ around the absorption edge we find $\rho(\epsilon)=n L \beta(\epsilon-$ $\Delta)^{2} /(b h c)$. As a function of energy relative to the absorption edge, this function vanishes in the same way as does the density of states for a three-dimensional photon gas or the density of states of massive particles in a three-dimensional harmonic trap. We demonstrate this behavior in Fig. 6. If the absorption profile close to the absorption edge vanishes faster than linearly, then the effective density of states vanishes faster than $(\epsilon-\Delta)^{2}$ and the integration over energy is still well behaved. Note that in the figure the region where $\rho(\epsilon) \propto(\epsilon-\Delta)^{2}$ is so close to the absorption edge that the dependence appears roughly linear visually.
[1] S. Zou, N. Janel, and G. C. Schatz, J. Chem. Phys. 120, 10871 (2004).

[2] F. J. Garcia de Abajo, Rev. Mod. Phys. 79, 1267 (2007).

[3] B. Auguié and W. L. Barnes, Phys. Rev. Lett. 101, 143902 (2008).

[4] I. Bloch, J. Dalibard, and W. Zwerger, Rev. Mod. Phys. 80, 885 (2008).

[5] P. Windpassinger and K. Sengstock, Rep. Prog. Phys. 76, 086401 (2013).

[6] P. Törmä and W. L. Barnes, arXiv:1405.1661.

[7] D. Jaksch, C. Bruder, J. I. Cirac, C. W. Gardiner, and P. Zoller, Phys. Rev. Lett. 81, 3108 (1998).

[8] M. Greiner, O. Mandel, T. Esslinger, T. W. Hänsch, and I. Bloch, Nature (London) 415, 39 (2002). .

[9] J. Kasprzak, M. Richard, S. Kundermann, A. Baas, P. Jeambrun, J. M. J. Keeling, F. M. Marchetti, M. H. Szymańska, R. André, J. L. Staehli et al., Nature (London) 443, 409 (2006).
[10] R. Balili, V. Hartwell, D. Snoke, L. Pfeiffer, and K. West, Science 316, 1007 (2007)

[11] J. D. Plumhof, T. Stöferle, L. Mai, U. Scherf, and R. F. Mahrt, Nat. Mater. 13, 247 (2014).

[12] J. Klaers, J. Schmitt, F. Vewinger, and M. Weitz, Nature (London) 468, 545 (2010).

[13] H. Yokoyama, Science 256, 66 (1992).

[14] P. R. Rice and H. J. Carmichael, Phys. Rev. A 50, 4318 (1994).

[15] I. Carusotto and C. Ciuti, Rev. Mod. Phys. 85, 299 (2013).

[16] R. F. Oulton, V. J. Sorger, T. Zentgraf, R.-M. Ma, C. Gladden, L. Dai, G. Bartal, and X. Zhang, Nature (London) 461, 629 (2009).

[17] W. Zhou, M. Dridi, J. Y. Suh, C. H. Kim, D. T. Co, M. R. Wasielewski, G. C. Schatz, and T. W. Odom, Nat. Nanotechnol. 8, 506 (2013).

[18] F. van Beijnum, P. J. van Veldhoven, E. J. Geluk, M. J. A. de Dood, G. W. 't Hooft, and M. P. van Exter, Phys. Rev. Lett. 110, 206802 (2013). 
[19] S. Rodriguez and J. Gómez Rivas, Opt. Express 21, 27411 (2013).

[20] A. I. Väkeväinen, R. J. Moerland, H. T. Rekola, A.-P. Eskelinen, J.-P. Martikainen, D.-H. Kim, and P. Törmä, Nano Lett. 14, 1721 (2014).

[21] L. Shi, T. K. Hakala, H. T. Rekola, J.-P. Martikainen, R. J. Moerland, and P. Törmä, Phys. Rev. Lett. 112, 153002 (2014).

[22] B. Fischer and R. Weill, Opt. Express 20, 26704 (2012).

[23] P. Kirton and J. Keeling, Phys. Rev. Lett. 111, 100404 (2013).

[24] Y. Yamamoto, S. Machida, and G. Björk, Phys. Rev. A 44, 657 (1991).

[25] M. Moskovits, Rev. Mod. Phys. 57, 783 (1985).

[26] L. Mandel and E. Wolf, Optical Coherence and Quantum Optics, 1st ed. (Cambridge University Press, Cambridge, 1995).
[27] G. Grynberg, A. Aspect, C. Fabre, and C. Cohen-Tannoudji, Introduction to Quantum Optics: From the Semi-classical Approach to Quantized Light (Cambridge University Press, Cambridge, 2010).

[28] J. Klaers, J. Schmitt, T. Damm, F. Vewinger, and M. Weitz, Appl. Phys. B: Lasers Opt. 105, 17 (2011).

[29] S. R. K. Rodriguez, A. Abass, B. Maes, O. T. A. Janssen, G. Vecchi, and J. Gómez Rivas, Phys. Rev. X 1, 021019 (2011).

[30] C. Pethick and H. Smith, Bose-Einstein Condensation in Dilute Gases (Cambridge University Press, Cambridge, 2008).

[31] J. Schmitt, T. Damm, D. Dung, F. Vewinger, J. Klaers, and M. Weitz, Phys. Rev. Lett. 112, 030401 (2014).

[32] E. Altman, J. Toner, L. M. Sieberer, S. Diehl, and L. Chen, arXiv:1311.0876. 